\title{
A search for p-modes and other variability in the binary system 85 Pegasi using MOST^ photometry
}

\author{
D. Huber ${ }^{1,2}$, J. M. Matthews ${ }^{3}$, B. Croll ${ }^{4}$, M. Obbrugger ${ }^{1}$, M. Gruberbauer ${ }^{1,5}$, D. B. Guenther ${ }^{5}$, W. W. Weiss ${ }^{1}$, \\ J. F. Rowe ${ }^{6}$, T. Kallinger ${ }^{1,3}$, R. Kuschnig ${ }^{1}$, A. L. Scholtz ${ }^{7}$, A. F. J. Moffat ${ }^{8}$, S. M. Rucinski ${ }^{4}$, \\ D. Sasselov ${ }^{9}$, and G. A. H. Walker ${ }^{10}$
}

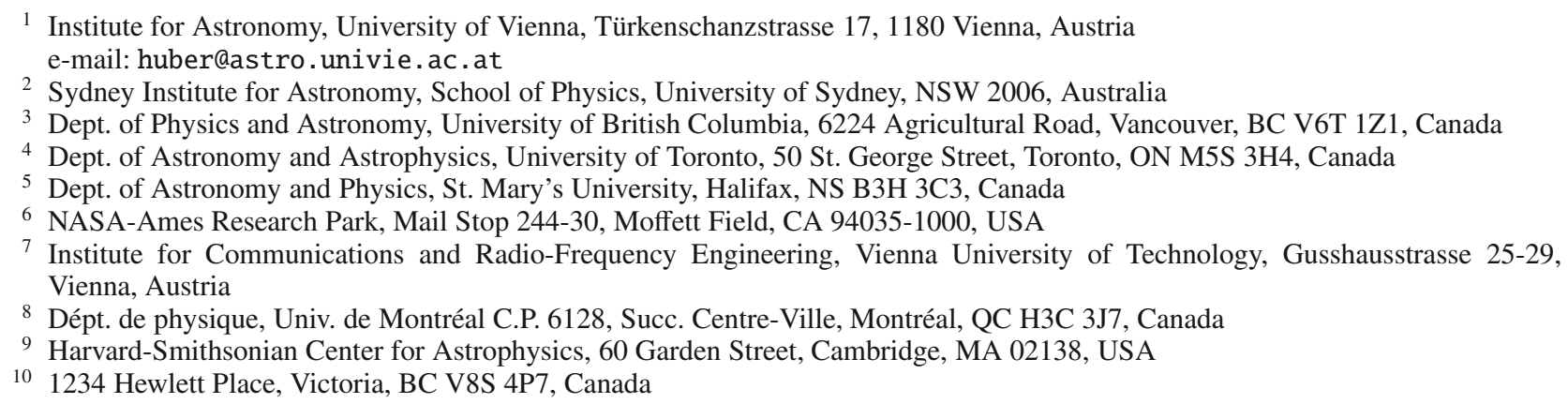

Received 24 March 2009 / Accepted 7 July 2009

\section{ABSTRACT}

\begin{abstract}
Context. Asteroseismology has great potential for the study of metal-poor stars due to its sensitivity to determine stellar ages. Solid detections of oscillation frequencies in stars with well constrained fundamental parameters, combined with a known rotation period, should significantly advance our understanding of stellar structure and evolution in context with metallicity effects.

Aims. Our goal was to detect p-mode oscillations in the metal-poor sub-dwarf 85 Peg A and to search for variability on longer timescales.

Methods. We have obtained continuous high-precision optical photometry of the binary system 85 Pegasi with the MOST (Microvariability \& Oscillations of STars) space telescope in two seasons (2005 \& 2007). The light curves were analyzed using traditional Fourier techniques. Furthermore, we redetermined $v \sin i$ for 85 Peg A using high resolution spectra obtained through the ESO archive, and used photometric spot modeling to interpret long periodic variations.

Results. Our frequency analysis yields no convincing evidence for p-modes significantly above a noise level of $4 \mathrm{ppm}$. Using simulated p-mode patterns we provide upper rms amplitude limits for 85 Peg A. After removal of instrumental trends the light curve shows evidence for variability with a period of about $11 \mathrm{~d}$ and this periodicity is also seen in the follow up run in 2007; however, as different methods to remove instrumental trends in the 2005 run yield vastly different results, the exact shape and periodicity of the 2005 variability remain uncertain. Our re-determined $v \sin i$ value for $85 \mathrm{Peg} \mathrm{A}$ is comparable to previous studies and we provide realistic uncertainties for this parameter. Using these values in combination with simple photometric spot models we are able to reconstruct the observed variations.

Conclusions. The null-detection of p-modes in $85 \mathrm{Peg} \mathrm{A}$ is consistent with theoretical values for pulsation amplitudes in this star. The detected long-periodic variation in the 85 Peg system must await confirmation by further observations with similar or better precision and long-term stability. If the $11 \mathrm{~d}$ periodicity is real, rotational modulation of surface features on one of the components is the most likely explanation.
\end{abstract}

Key words. techniques: photometric - stars: individual: 85 Peg - stars: oscillations - stars: rotation - starspots - binaries: general

\section{Introduction}

With observations dating back to the mid-19th century by Baily (1845), 85 Pegasi (HD 224930) is a very well studied visual binary system with an angular separation of 0.83 arcsec. Due to its proximity $(d=12 \mathrm{pc})$ and brightness $(V=5.75)$, the orbital elements have been determined to high accuracy through photometry and spectroscopy. Griffin (2004) provides a comprehensive review of the wealth of data obtained for the 85 Peg system, including a spectroscopic orbital solution with an orbital period

\footnotetext{
* Based on data from the MOST satellite, a Canadian Space Agency mission, jointly operated by Dynacon Inc., the University of Toronto Institute for Aerospace Studies and the University of British Columbia, with the assistance of the University of Vienna.
}

of 26.31 years and a total mass of $1.49 M_{\odot}$. The dynamically measured relative masses of the binary system, however, indicating a secondary equally or only slightly less massive than 85 Peg A, disagree with the observed magnitude difference of $\Delta m_{\mathrm{V}} \simeq 3$ between the components. It was suggested as early as 1948 by Hall (1948) that the secondary star in 85 Peg may be itself a binary system. Evolutionary models by Fernandes et al. (2002), D’Antona et al. (2005) and Bach et al. (2007) all support the binarity of $85 \mathrm{Peg} \mathrm{B}$.

The primary $85 \mathrm{Peg} \mathrm{A}$ is metal-poor, with measurements of $[\mathrm{Fe} / \mathrm{H}]=-0.88$ (Allende Prieto et al. 2004), -0.78 (Holmberg et al. 2007) and $[\mathrm{M} / \mathrm{H}]=-0.69$ (van't Veer 2000; cf. Fernandes et al. 2002). The space velocity of the star, along with its low 
Table 1. Main parameters of $85 \mathrm{Peg}$ A and B compiled from the literature.

\begin{tabular}{cccc}
\hline \hline Parameter & 85 Peg A & 85 Peg B [a,b $]^{i}$ & Ref $^{i i}$ \\
\hline$V$ (mag) & $5.81 \pm 0.03$ & $8.89 \pm 0.29$ & 1 \\
Sp.Type & G5 IV & K6-8 V & 2 \\
$M\left(M_{\odot}\right)$ & $0.77 \pm 0.05$ & $0.72 \pm 0.05[0.5,0.2]$ & 3,4 \\
$L\left(L_{\odot}\right)$ & $0.617 \pm 0.02$ & $0.072 \pm 0.03[0.064,0.0085]$ & 5 \\
$T_{\text {eff }}(\mathrm{K})$ & $5600 \pm 50$ & $4200 \pm 200$ & 6,7 \\
$\log g$ & $4.6 \pm 0.1$ & $4.8 \pm 0.2$ & 4,7 \\
{$[\mathrm{Fe} / \mathrm{H}]$} & -0.78 & $\ldots$ & 8 \\
Age $(\mathrm{Gyr})$ & $9.3 \pm 0.5$ & $\ldots$ & 5 \\
\hline
\end{tabular}

${ }^{i}$ Numbers in brackets are suggested values by D'Antona et al. (2005) and Bach et al. (2007) for the suspected binary $85 \mathrm{Peg} \mathrm{Ba}$ and Bb.

${ }^{i i}$ Reference coding: (1) ten Brummelaar et al. (2000), (2) Fulbright (2000), (3) Griffin (2004), (4) Bach et al. (2007), (5) D'Antona et al. (2005), (6) Fernandes et al. (2002), (7) van't Veer et al. (2005); cf. D'Antona et al. (2005), (8) Holmberg et al. (2007).

metallicity, qualify it as a sub-dwarf. 85 Peg A was classified as a G5 sub-dwarf by Fulbright (2000). Based on only the visual magnitude difference (neglecting the contradicting mass ratio from the orbital solution), $85 \mathrm{Peg} \mathrm{B}$ is usually assumed to be a K6-8 dwarf.

Fernandes et al. (2002) modeled the initial abundances, age and mixing-length parameters of $85 \mathrm{Peg} \mathrm{A}$ and estimated an age of $9.3 \pm 0.5$ Gyr. Using effective temperatures derived from spectroscopy and the measured Hipparcos parallax, D'Antona et al. (2005) computed evolutionary tracks for a set of possible metallicities and ages of the system. Their evolutionary models allow ages between about 8 and 14 Gyr. They generated pulsational eigenspectra from their models, predicting that solar-like p-modes of low degree $(\ell=0-3)$ in 85 Peg A could occur across a frequency range of $1-5 \mathrm{mHz}\left(\simeq 80-400 \mathrm{~d}^{-1}\right)$. Table 1 lists all important parameters of the binary system 85 Peg which are relevant for this paper.

The detection of oscillations in stars like $85 \mathrm{Peg} \mathrm{A}$ is particularly interesting due to the sensitivity of asteroseismology to determine stellar ages. While the small frequency separation is the more sensitive observable for this purpose, even a measurement of the large separation considerably constrains the parameter space which often is more uncertain for metal-poor objects than for other stars. The first successful application of asteroseismology to constrain the age of a metal-poor star has been performed by Bedding et al. (2006) using Doppler velocity observations of the subgiant $v$ Indi $([\mathrm{Fe} / \mathrm{H}]=-1.4)$. Even before that, Mosser et al. (2005) detected oscillations in velocity measurements of HD $49933([\mathrm{Fe} / \mathrm{H}]=-0.4)$, which were later confirmed photometrically by the CoRoT space telescope (Appourchaux et al. 2008; Michel et al. 2008). The rich oscillation spectrum of HD 49933 has recently been subject to some discussion, in particular in context with its fundamental parameters (Bruntt et al. 2008; Ryabchikova et al. 2009; Bruntt 2009) and the ability to model the oscillation frequencies (Kallinger et al. 2008a), underlining the importance of studying stellar oscillations in metalpoor objects with well constrained parameters. Further recent detections of solar-like oscillations in metal-poor stars include the CoRoT time series of HD $181906([\mathrm{Fe} / \mathrm{H}]=-0.1)$ (Michel et al. 2008) and Doppler measurements of HD 203608 (Mosser et al. 2008) $([\mathrm{Fe} / \mathrm{H}]=-0.65)$.

The detection of p-modes and the measurement of the large and small frequency spacings in the high-overtone eigenspectrum of 85 Peg A would put important constraints on its mass and age. If the star were as old as 12 Gyr or more, this would also place a meaningful lower limit on the age of the Galaxy. For these reasons, even before the work of Fernandes et al. (2002) and D'Antona et al. (2005), 85 Peg was selected as a primary science target for the MOST space mission, to search for $\mathrm{p}$-modes in the metal-poor sub-dwarf component of the binary. The continuous time coverage, high sampling rate and high photometric precision of the MOST photometry also make the data suited to search for other variability in the binary system.

\section{MOST observations and data reduction}

The MOST (Microvariability \& Oscillations of STars) space mission is the first satellite designed to specifically conduct asteroseismic observations of stars (Walker et al. 2003; Matthews 2007). Equipped with a $15 \mathrm{~cm}$ Rumak-Maksutov telescope feeding a CCD photometer through a custom broadband optical filter (350-700 nm), MOST can collect rapid high-precision photometry of bright stars with nearly uninterrupted coverage for up to 2 months.

Depending on the brightness of the target of interest, MOST uses two different observing modes. In the primary mode, the target starlight enters a field stop about 1 arcmin in diameter and illuminates an extended fixed image of the telescope pupil on the science CCD detector using a Fabry microlens. For fainter stars, direct photometry of stars is obtained corresponding to traditional CCD photometry with a FWHM of about 2-3 pixels. With a pixel scale of $\sim 3$ arcsec, the binary nature of 85 Peg remains unresolved (the integrated signal, however, being dominated by 85 Peg A which is about $16 \times$ brighter than its companion based on the observed visual magnitude difference).

MOST observed 85 Peg in 2005 and 2007. In 2005, the visual brightness limit for the primary observing mode was $V \sim 6$, making $85 \mathrm{Peg}(V=5.75)$ the faintest star ever observed using Fabry imaging. In early 2006, the satellite tracking CCD stopped functioning, most likely due to a strong particle hit. Consequently, guide star observations had to be relocated to the science $\mathrm{CCD}$, limiting the exposure times for the program stars and constraining the brightness limit for Fabry Imaging. Therefore, $85 \mathrm{Peg}$ was observed using direct imaging in the follow-up run in 2007.

For both observation modes, data reduction pipelines have been developed and are fully described by Reegen et al. (2006) for Fabry imaging and Rowe et al. (2006) as well as Huber \& Reegen (2008) for direct imaging. The main issue in MOST data reduction is the removal of stray light influences from the data. In all programs, the concept of decorrelation is used which is based on the comparison of intensities originating from the star with intensities of background readings. The $85 \mathrm{Peg}$ datasets were reduced according to the routines described by Reegen et al. (2006) and Huber \& Reegen (2008).

85 Peg was observed by MOST in 2005 for 25.6 days with almost no interruptions. The exposure time was $50 \mathrm{~s}$ with a sampling interval of $55 \mathrm{~s}$. After outlier corrections in the reduction pipeline the final light curve consisted of 34323 measurements in total. The reduced light curve shows very good quality with point-to-point scatter of $0.48 \mathrm{mmag}$, making it well suited to search for p-mode oscillations. In 2007, MOST returned to $85 \mathrm{Peg}$ for another run spanning a total of 25.5 days, this time with observations being performed in direct imaging mode with considerably shorter exposure times and images mostly being obtained every fifth MOST orbit. Due to the more difficult thermal conditions caused by target switching, the data are influenced by strong instrumental trends, which in particular affected the beginning and end of the run. Therefore, only the central part 
Table 2. Observation log of the MOST 85 Peg runs in 2005 and 2007.

\begin{tabular}{lcc}
\hline \hline Observation run & 2005 & 2007 \\
\hline Start date (HJD-2 451545) & 2085.43 & 2830.81 \\
End date (HJD-2 451 545) & 2111.01 & 2842.50 \\
Total obs. time (d) & 25.58 & 11.69 \\
Number of exposures & 34323 & 5738 \\
Duty cycle (\%) & 85 & 14 \\
Exposure time (s) & 50 & $13.25[53]^{i}$ \\
Sampling time (s) & 55 & $25[100]$ \\
Point-to-point scatter (mmag) & 0.48 & $0.83[0.51]$ \\
\hline
\end{tabular}

${ }^{i}$ Values in brackets for the 2007 run correspond to the light curve with 4 datapoint bins (to make the scatter comparable to the 2005 run).

of the light curve spanning over about 12 days was used for the analysis. The data also show a slightly higher scatter than the earlier run, with an increased point-to-point spread of the light curve binned to about the same exposure time as the 2005 data. An observation log for both runs is given in Table 2 .

\section{The search for p-modes}

\subsection{Frequency analysis}

Due to the data quality difference, the search for p-modes was only performed in the 2005 data, using Fourier analysis and least squares fitting. The significances of identified peaks were estimated with SIGSPEC (Reegen 2007) which, based on a falsealarm probability, incorporates frequency, amplitude and phase information into the calculation. Significant frequencies are identified through consecutive prewhitening and least squares fits. The analysis was performed up to the Nyquist frequency of the data set $\left(v_{\mathrm{Nyq}} \simeq 9 \mathrm{mHz} \simeq 780 \mathrm{~d}^{-1}\right)$.

Figure 1 shows the Fourier amplitude spectrum in the frequency region where $\mathrm{p}$-modes have been predicted. The amplitudes have been normalized by $N / 2$ where $N$ is the number of datapoints in the light curve. The largest peaks in the amplitude spectrum are residuals of the background variation at the orbital frequency of the MOST satellite $\left(164 \mu \mathrm{Hz}=14.19 \mathrm{~d}^{-1}\right)$ and its harmonics, marked in Fig. 1 by vertically dotted lines. Note that even the largest of these has an amplitude of only about $35 \mathrm{ppm}$ (0.035 mmag), and above a frequency of $2.5 \mathrm{mHz}$, there is only one with an amplitude as high as $15 \mathrm{ppm}$. There is also some power in sidelobes of the harmonics of the satellite orbital frequency, spaced by 1 and $2 \mathrm{~d}^{-1}$, due to the daily modulation of scattered earthshine.

Aside from these instrumental frequencies, there is no apparent power excess above the noise level visible in the spectrum. The SIGSPEC analysis shows that, above $0.57 \mathrm{mHz}\left(50 \mathrm{~d}^{-1}\right)$, there are only two peaks (not associated with MOST orbital harmonics or $1 \mathrm{~d}^{-1}$ sidelobes) with a significance greater than 4 (corresponding to an amplitude $S / N$ of about 3.5). One of these peaks can be seen in Fig. 1 near $2.55 \mathrm{mHz}$.

\subsection{Estimation of the detection limit}

The MOST observations constitute the first large-scale dedicated observing effort to detect $\mathrm{p}$-mode oscillations in the 85 Peg system. Despite the null-result, the data should allow us to draw valuable conclusions for future efforts by setting firm limits on the possible pulsation properties of $85 \mathrm{Peg} \mathrm{A}$.

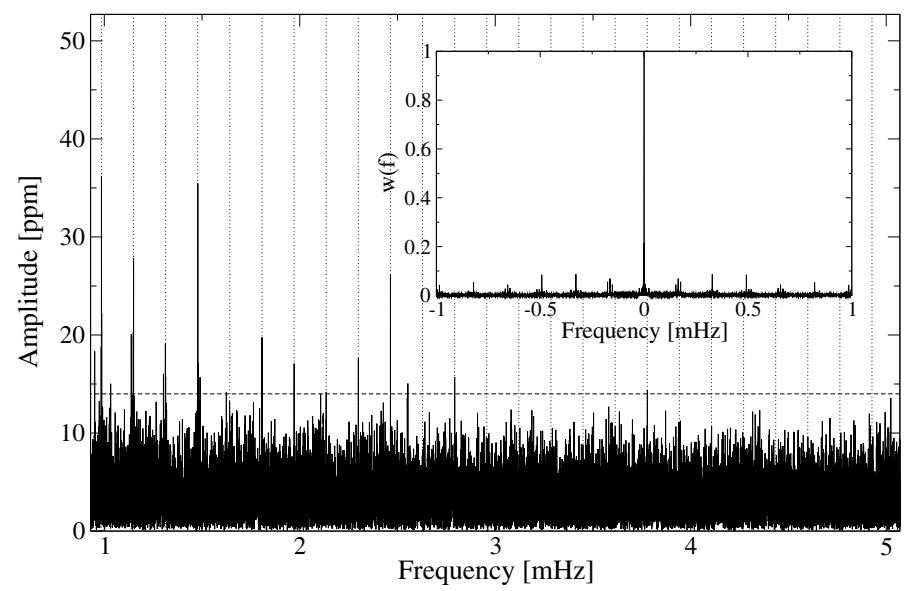

Fig. 1. Fourier amplitude spectrum of the 2005 MOST light curve of 85 Peg in the frequency region where p-modes have been predicted. Vertical dotted lines correspond to harmonics of the orbital frequency of the MOST satellite. The horizontal dashed line shows the $3.5 \sigma$ detection limit (corresponding roughly to a spectral significance of 4 ) for the noise level in this frequency region. The insert displays the window function of the light curve.

Considering the average noise level of $4 \mathrm{ppm}$ in the amplitude spectra at a frequency range where the p-modes are predicted by theory, we can set an upper limit of pulsation in the MOST observations in Fourier domain to about 14-18 ppm, assuming a $3.5-4.5 \sigma$ detection limit. Simulations of 11 coherent sinusoidal variations inserted into the MOST photometry spaced by $\Delta v=170 \mu \mathrm{Hz}$ (a value lying in the range of the models calculated by D'Antona et al. 2005) and centered around $3.4 \mathrm{mHz}$ (using the relation of Kjeldsen \& Bedding 1995, for the estimated mass, radius and effective temperature of 85 Peg A) showed that peaks with amplitudes of $15 \mathrm{ppm}$ are securely recovered using the same approach as in the real data using SigSPEC. Note that this limit is only valid under the assumption that the mode lifetimes are longer than the observation timebase and therefore can be modeled as coherent sinusoidal variations.

Going one step further, we have also included the nature of the pulsation in the simulations. It is widely accepted that p-mode oscillations in sun-like stars are driven by turbulent motions in the convective layers in the stars, causing a stochastic excitation and subsequent damping of the pulsation modes rather than a coherent oscillation. It has been shown that the lifetimes of the oscillations for sun-like stars are very short, i.e. on the order of a couple of days (e.g. for $\alpha$ Cen A, see Bedding et al. 2004), while both short as well as substantially longer lifetimes (i.e. on the order of weeks or months) have been detected in giant and subgiant stars (Stello et al. 2006; Carrier et al. 2007; Kallinger et al. 2008b). While the situation of subdwarf stars remains undetermined, it seems appropriate from these results that with a dataset as long as the MOST observations the effects of stochastically driven pulsation should not be neglected.

The main effect when analyzing such signals in the Fourier domain is that the oscillation will not be described purely by the window function of the dataset, but rather by a series of peaks scattered around the true frequency value. The shape of this distribution is commonly described by a Lorentzian profile with the FWHM of the profile being correlated to the mode lifetime and the height, in combination with the width, being related to the total energy of the pulsation mode (Houdek 2006). Hence, the resulting amplitude of a mode in a Fourier spectrum is not only simply dependent on the maximum displacement in the time 
series, but also on the amount of damping and the resulting mean variation in the data set. As given by Baudin et al. (2005) and Barban et al. (2007), the mean mode amplitude $A$ can be expressed as

$A^{2}=\frac{T}{4 \tau} H=\frac{1}{4 \tau} \mathcal{H}$,

with $T$ denoting the effective length of the dataset, $\tau$ is the mode lifetime, $H$ the height of the Lorentzian profile in power $\left(\mathrm{ppm}^{2}\right)$ and $\mathcal{H}$ the height of the Lorentzian profile in power density $\left(\mathrm{ppm}^{2} \mathrm{~Hz}^{-1}\right)^{1}$. The effective dataset length $T$ is evaluated as the reciprocal value of the area under the spectral window (in power), and in the case of the MOST $85 \mathrm{Peg}$ observations gives $T=1.8895 \mathrm{Msec}=21.869 \mathrm{~d}$.

To set limits on the mean mode amplitude for 85 Peg A, we simulated solar-like oscillations implementing the concept by Chaplin et al. (1997) adapted to the sampling of the MOST 85 Peg 2005 time series. Using the same input frequencies as in the simulations containing coherent signal as described above, about 1000 datasets were generated for mode lifetimes ranging from 1-26 $\mathrm{d}$ in steps of $1 \mathrm{~d}$ and for mean amplitudes from $1-40 \mathrm{ppm}$ in steps of $1 \mathrm{ppm}$. Note that in order to keep the computation time reasonable and to solely study the effects of different amplitudes and mode lifetimes, each simulation was computed as a single realization (i.e. the stochastic excitation/damping pattern was the same for each simulated data set). The mean mode amplitude (which was set to the same value for all frequencies) was simulated by setting the time-domain standard deviation of a single realization to a fixed value $A_{\text {rms }}$. Note that this value is not the same as the maximum peak height in the resulting Fourier spectrum, which is a priori unknown. In the following, the standard deviation of the variation in the time domain will be denoted rms amplitude, while the resulting peak height in the Fourier spectrum will be referred to as Fourier amplitude. For each simulation, the mean $S / N$ ratio of the six highest peaks was calculated (i.e., we assumed 6 out of 11 frequencies to be a successful detection of p-mode pulsation). The results of the simulations are shown in Fig. 2.

Not surprisingly, the simulations show that oscillations with larger amplitudes and longer mode lifetimes would be easier to detect. For example, an rms amplitude of $16 \mathrm{ppm}$ of the oscillations would remain undetected if the mode lifetimes are very short, even though Fourier domain noise would in principle allow a $4 \sigma$ detection. Only for lifetimes getting closer to the length of the dataset are the expected $S / N$ ratios as known for a coherent signal fulfilled (e.g., a coherent sinusoidal variation with a rms amplitude of $15 \mathrm{ppm}$ produces a peak amplitude of about $20 \mathrm{ppm}$, corresponding to a $S / N \sim 5$ in the $85 \mathrm{Peg}$ data). The positive influence of longer lifetimes, however, decreases rapidly as the lifetime approaches the length of the dataset, reaching a detection level of about $10 \mathrm{ppm}$ at $\tau>10 \mathrm{~d}$. Note that in Eq. (1), $H$ is only representative of the power excess height for large values of $T / \tau$, i.e. when the Lorentzian profile is well resolved. This explains why a signal formally described by, e.g., $H=18 \mathrm{ppm}$ can remain undetected for long lifetimes (i.e. small $T / \tau$ ) in the MOST photometry simulations. With this in mind, the following conclusions on the oscillation properties of 85 Peg can be drawn: 1) independent of the amplitude or the mode lifetime of the oscillations, the height of the Lorentzian profiles must be lower than about $20 \mathrm{ppm}$ to remain undetected in the MOST data; 2) if the

\footnotetext{
1 Note that this relation is only valid when the amplitude spectrum is normalized by $N / 2$, where $N$ is the number of datapoints in the time series.
}

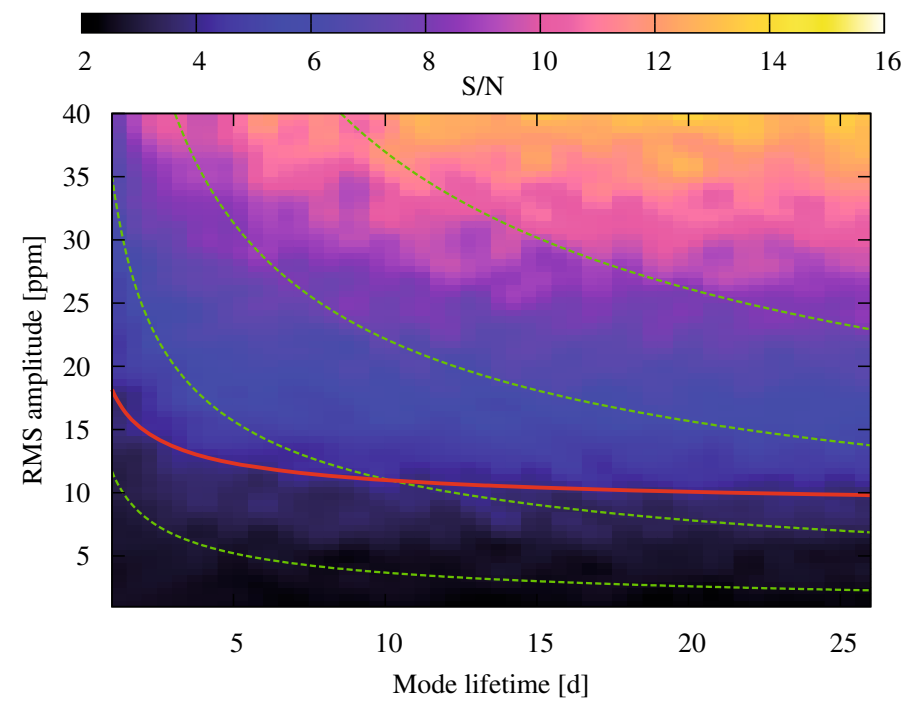

Fig. 2. Signal-to-Noise ratio of the six highest peaks of simulated solarlike pulsation using the MOST $85 \mathrm{Peg}$ data as a function of mode lifetime and rms amplitude in the time domain. Points of constant Lorentzian profile height $H$ are shown as dashed green lines (from the top right to the bottom left: $50 \mathrm{ppm}, 30 \mathrm{ppm}, 15 \mathrm{ppm}$ and $5 \mathrm{ppm}$ ). The thick solid red line shows a power law fit to bins of data points with $S / N=3.5-4.5$.

mode lifetimes in 85 Peg are very short ( $\sim$ days $)$, the rms amplitude of the signal must lie below a level of about $12-18 \mathrm{ppm}$. If the lifetimes are long ( $\sim$ weeks $)$, this limit lies at approximately $10 \mathrm{ppm}$.

To predict upper limits for the height of the Lorentzian profiles for 85 Peg A which are independent of the observation timebase, we converted the values derived from the simulations into power density. The top panel of Fig. 4 shows, for each mode lifetime bin of the simulations, data points with $S / N=3.5-4.5$ and the corresponding power law fit in power density. Combined with the Fourier amplitude limit of $\sim 16$ ppm set by MOST, this provides firm limits on the oscillation properties of $85 \mathrm{Peg} \mathrm{A}$ and could be used as a prior information to plan and analyze further observations with the goal of detecting solar-like oscillations in this star.

\subsection{Comparison with theoretical values}

In order to compare our upper limits on the oscillation amplitudes with predictions, we follow the method by Michel et al. (2009) to calculate bolometric amplitudes, which are independent of the instrument being used. Using the transmission curve of the MOST filter and an effective temperature of $5600 \mathrm{~K}$ for 85 Peg A, we calculate a response function for radial modes of $R_{0}=4.787$. Following the convention by Michel et al. (2009), we then convert our upper rms amplitude limits as derived in the previous section into bolometric amplitudes per radial mode as

$A_{\mathrm{bol}, \mathrm{l}=0}=\frac{4}{R_{0}}\left(\frac{\delta I}{\bar{I}}\right)(t)$

where the last term denotes the observed rms intensity fluctuations, which we identify as our definition of the rms amplitude.

Theoretical amplitudes calibrated to observations indicate that bolometric photometric amplitudes scale as $(L / M)^{\alpha} T_{\text {eff }}^{-1}$ (Kjeldsen \& Bedding 1995), with numerical simulations suggesting a value of $\alpha \sim 0.7$ (Samadi et al. 2007). Using the solar 


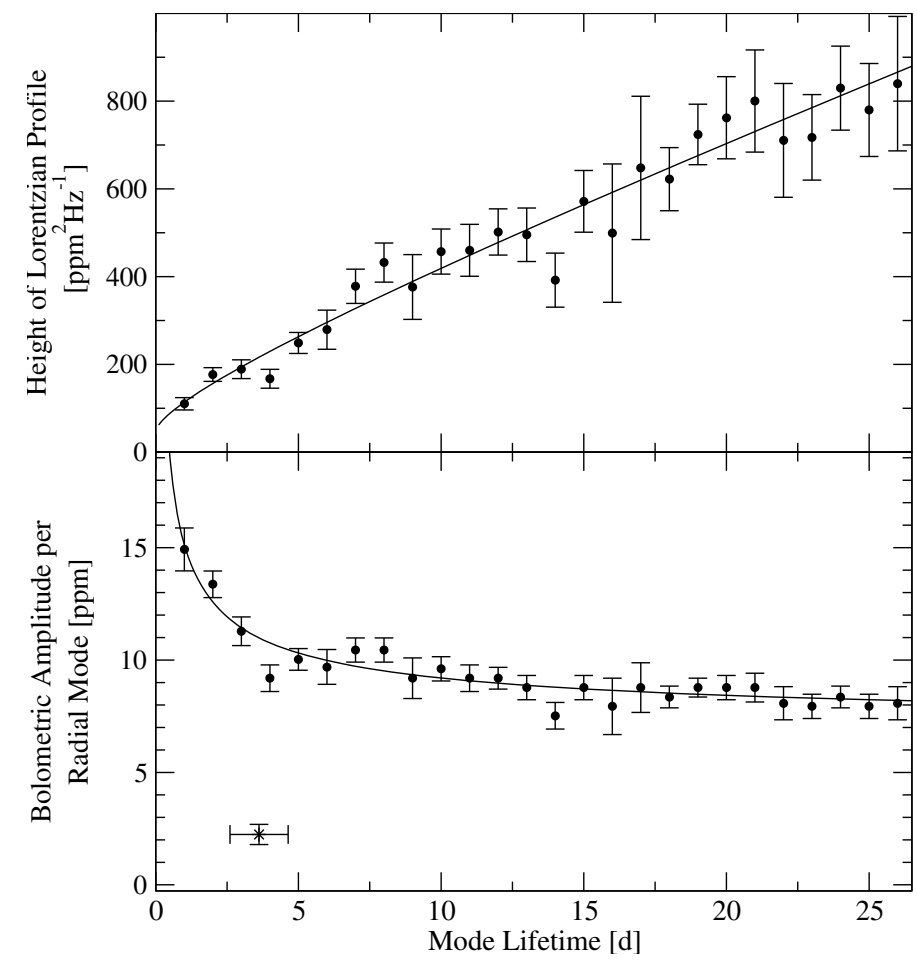

Fig. 3. Upper limits on oscillation properties of $85 \mathrm{Peg} \mathrm{A}$ as a function of mode lifetime, based on simulations using MOST photometry. Top panel: upper limit on the height of the Lorentzian profile in power density. Bottom panel: upper limit on the bolometric amplitude per radial mode. The star symbol shows the expected value based on theoretical scaling relations with $3 \sigma$ error bars.

reference value of $2.53 \pm 0.11 \mathrm{ppm}$ for $A_{\mathrm{bol}, 1=0}$ by Michel et al. (2009), the mass, luminosity and effective temperature (with a more conservative error of $100 \mathrm{~K}$ ) of 85 Peg A yield an expected theoretical amplitude of $2.2 \pm 0.2 \mathrm{ppm}$. As an estimate for the expected mode lifetime we use the recently suggested scaling relation by Chaplin et al. (2009) proposing $\tau \propto T_{\text {eff }}^{-4}$, and with a solar reference value of $\tau_{\odot}=3.2 \pm 0.2 \mathrm{~d}$ (Chaplin et al. 2005) arrive at $\tau=3.6 \pm 0.3 \mathrm{~d}$ for the expected mode lifetime of $85 \mathrm{Peg} \mathrm{A}$.

The bottom panel of Fig. 3 shows the upper limits for 85 Peg A as derived from the simulations converted to bolometric amplitudes per radial mode, compared to the theoretical values. Clearly, the low value for the theoretical amplitude would in any case remain undetected in the MOST 85 Peg data. This is also true for the mean velocity amplitude of $9 \mathrm{~cm} \mathrm{~s}^{-1}$ estimated by Houdek (cf. D'Antona et al. 2005) for a model of 85 Peg A, which, using the relation by Kjeldsen \& Bedding (1995), would translate in a bolometric luminosity amplitude of $\sim 1 \mathrm{ppm}$. Considering these estimated values, we are not able to draw any conclusions about the mode lifetimes for this star and can conclude that our results as upper limits are in agreement with predictions.

\subsection{Solar-type oscillations or wishful thinking?}

In a further investigation of the 2005 data, a relaxed spectral significance limit of 3 (corresponding to a $S / N$ of about 3 ) was used to search for p-modes in the 85 Peg data. In this case, SIGSPEC identified several peaks which were mostly located in two distinct frequency regions, one reaching from $1-3 \mathrm{mHz}$, the other from about $5-7 \mathrm{mHz}$. Eliminating all signals which are due to known instrumental artifacts and considering only values in the

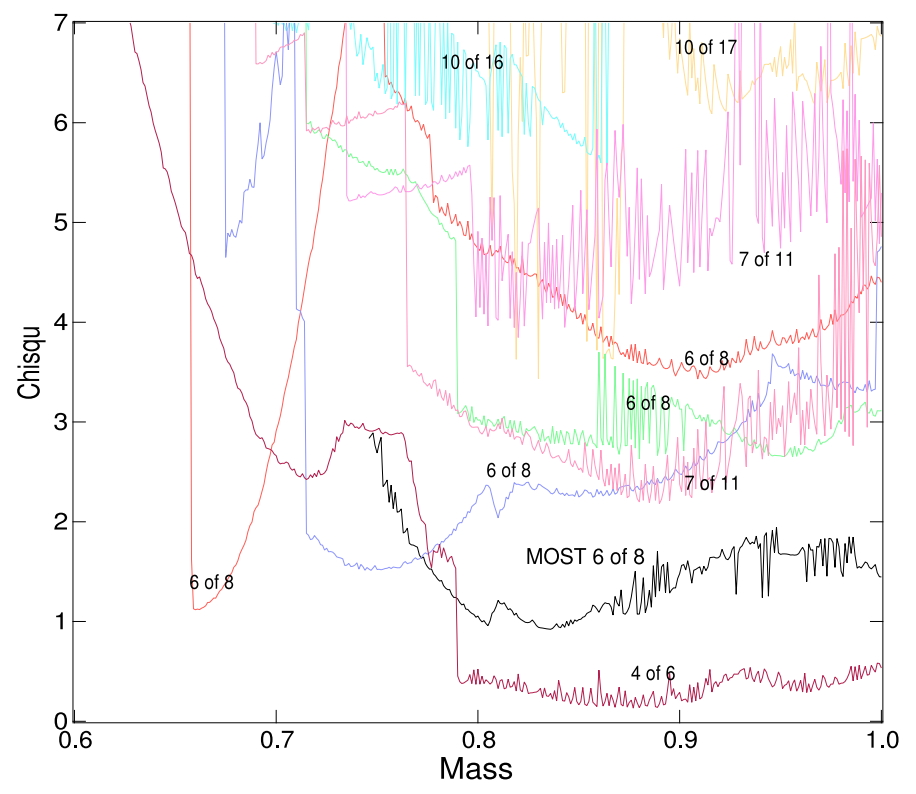

Fig. 4. Model fitting results as a function of mass using frequencies extracted from randomly generated datasets with a limit of $S / N \geq 3$. Line labels indicate the number of frequencies out of the total number of detected frequencies in a given dataset used for the fit. The fitting result using the real MOST dataset is also shown.

frequency range where p-modes are predicted, these frequency candidates were fitted to a dense grid of 300000 stellar models (Guenther et al. 1992). The quality of the fit was evaluated by a $\chi^{2}$ test, with a value of $\chi^{2} \leq 1$ meaning that, on average, the model frequencies that are used in the fit are within the uncertainties of the observed frequencies (see Guenther \& Brown 2004, for details). This attempt resulted in a model fitting to about $65 \%$ of the observed frequencies with a $\chi^{2} \leq 1$. This model is in agreement with the previously estimated position of 85 Peg A in the HR-Diagram, with a large frequency separation of $172 \mu \mathrm{Hz}$.

To test the credibility of this model fit, we generated 8 simulated datasets, 4 using Gaussian distributed random numbers, the other half by randomly shuffling the 2005 light curve. In both cases the same sampling, frequency analysis and model fitting procedure as for the real light curve was applied. The results of this test are shown in Fig. 4, with the fit quality $\chi^{2}$ given as a function of mass. As the figure shows, there is indeed one random dataset where frequencies could be extracted that also fit with a $\chi^{2} \leq 1$, indicating a similar fit quality as the best model derived for the real data.

The result of this test underlines that when searching for low-amplitude p-mode oscillations, it is extremely important to discern real evidence from wishful thinking. In a recent analysis of MOST data on Procyon, Guenther et al. (2008) have demonstrated the risks when searching for comb-like structures in power spectra of sun-like stars using a priori assumptions. The test presented here is yet another example for this, showing that a $S / N$ limit of 3 in amplitude (hence $S / N \geq 9$ in power, which is more widely used) is not sufficient to distinguish real signal from peaks generated from random noise, and can lead to misleading results.

\section{Long periodic variability}

Although designed as a non-differential photometer, the MOST space telescope has proven to be remarkably stable over long 
timescales, making it also suited to search for long-periodic variability. Instrumental trends, if present, can in many cases be identified, reconstructed and corrected by analyzing the satellites telemetry data such as board or preamplifier temperature.

The 2005 light curve of 85 Peg shows a dominant long-term instrumental trend which is confirmed by inspection of both the board and preamplifier temperature data. All three datasets are displayed in panels (a), (b) and (c) of Fig. 5. In a first attempt to correct the trend seen in panel (a), we fitted a polynomial function to the data (dashed line). The residuals of this fit, low-pass filtered to remove residual variability with periods of one day and averaged into bins of length $101 \mathrm{~min}$ (about one MOST orbital period), show two cycles of a variability with a period of about $11 \mathrm{~d}$ and an amplitude of $0.3 \mathrm{mmag}$ (panel (d) of Fig. 5). The 2007 data, which showed a less complex instrumental trend corrected by a simple linear fit, seems to confirm this variability detected in 2005, showing one full cycle of a light variation with a comparable period and amplitude (Fig. 5, panel (f)). Combining the 2005 and 2007 data, a Fourier analysis yields a best fit period of $11.6 \mathrm{~d}$ for the variation.

In a later stage of the analysis, an attempt was made to correct the 2005 data trend by directly using and fitting the variations seen in the satellite telemetry data ${ }^{2}$. For this purpose, the telemetry data were binned to two orbital period means and heavily smoothed, so only long-periodic features remain present. These smoothed versions (shown as solid lines in panels (b) and (c) of Fig. 5) were then correlated to the light curve in two segments of the run, and using the comparison set showing the highest correlation coefficient (in both cases the preamplifier temperature, see panel (b) of Fig. 5) all correlations were removed using linear regressions. The resulting fit which was subtracted is shown as a solid line in panel (a) of Fig. 5. The resulting light curve (panel (e) in Fig. 5) shows a much more complex behaviour, with the first cycle of the $11 \mathrm{~d}$ variability almost entirely removed.

The considerable discrepancy between the results of the two background removal methods is obviously alarming. However, as the variability of the first reduction method was confirmed in the 2007 dataset, we feel that we have observed the $\sim 11 \mathrm{~d}$ periodicity in both epochs of data. We stress that without follow-up observations to confirm the observed variability we cannot rule out the possibility that the correlation between the 2005 and 2007 epochs of data is purely coincidental, and that the variability changed considerably between 2005 and 2007 as illustrated with our second reduction method. It must be noted that the usage of the decorrelation technique in this case assumes a linear relationship of temperature and intensity variability, which might be an oversimplification of the problem. Indeed recent results on the low frequency variability of the MOST data on Procyon has shown evidence that telemetry data decorrelation seems to overcorrect existing intrinsic variability (Huber et al., in preparation). Thus we feel that the most likely possibility is that we have observed a common $\sim 11 \mathrm{~d}$ variability in our two epochs of data. In the following sections we present attempts to interpret the variability assuming that the 2005 light curve in panel (d) of Fig. 5 is indeed intrinsic.

The variability could originate in either of the two stars in the binary, since the MOST photometry does not resolve the individual components. If it is due to star A, then the amplitude of the variability is $0.3 \mathrm{mmag}$. If it is due to star $\mathrm{B}$, then the amplitude

\footnotetext{
${ }^{2}$ Note that the brightest star simultaneously observed in the 2005 run is $\sim 3$ mag fainter and hence no comparison stars can be used for this correction.
}
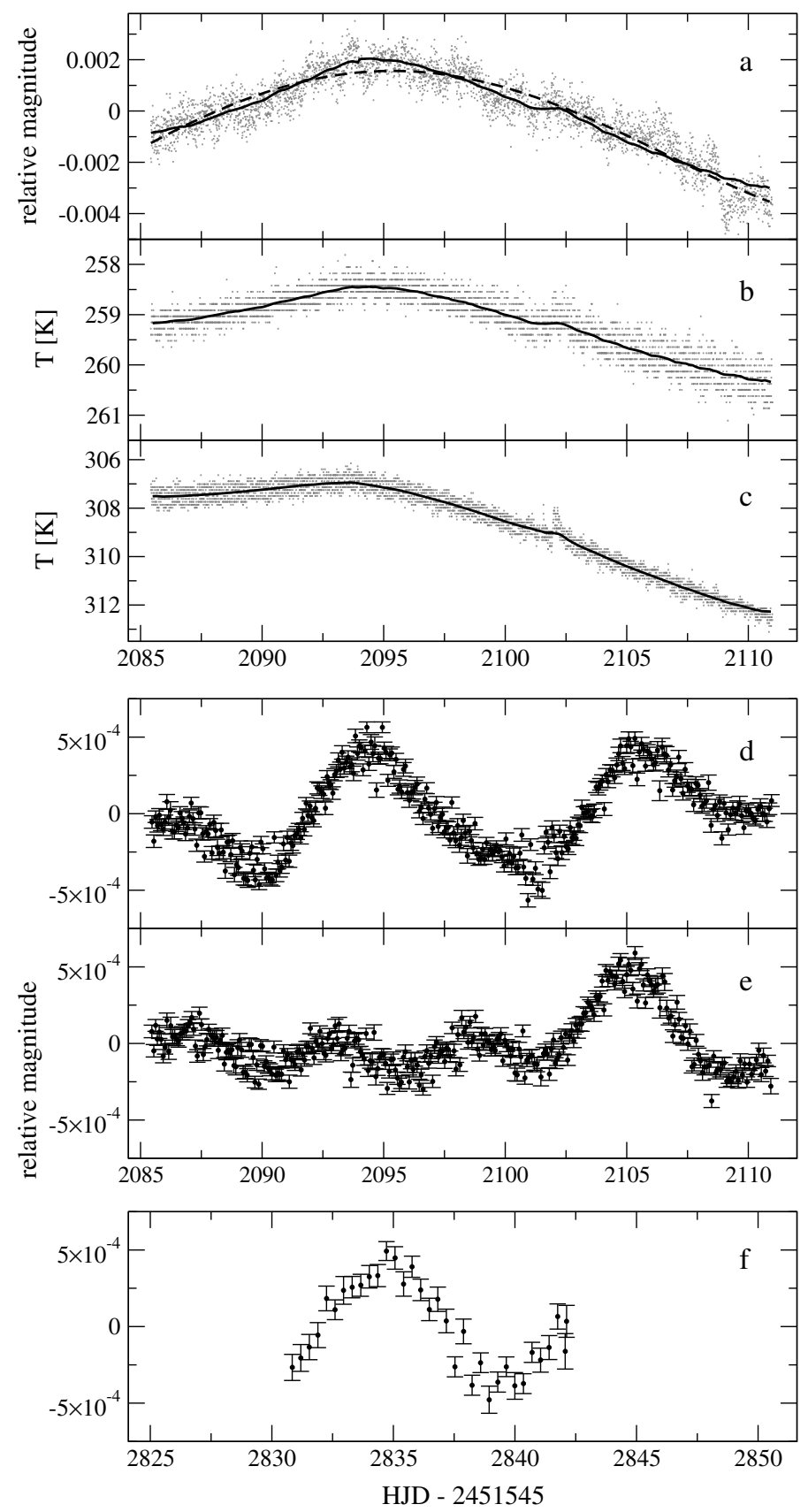

Fig. 5. Panel a): original 200585 Peg light curve (every tenth data point is shown) together with different fits used to correct the instrumental trend (see text). Panel b): satellite preamplifier temperature curve (every tenth data point is shown). The solid line shows a smoothed version. Panel c): same as panel b) but for satellite board temperature. Panel d): binned and low-pass filtered 200585 Peg light curve derived by subtracting a polynomial fit (dashed line in panel a)). Panel e): same as in panel d), but derived by decorrelating variations found in the satellite telemetry data (solid line in figure panel a)). Panel f): corrected 2007 85 Peg light curve. Note that the y-axis scale for panels d)-f) is the same, but differs from the scale in panel a).

we observe has been diluted significantly by the light of star A. The intrinsic amplitude would be approximately $5 \mathrm{mmag}$, based on the visual magnitude difference of 85 Peg A and 85 Peg B.

We have considered two possible explanations of the periodicity: (1) light effects due to the possible binarity of component $\mathrm{B}$, and (2) rotational modulation of the light of either star A or B due to surface spots. 


\subsection{Binarity of $85 \mathrm{Peg} B$}

85 Peg B has long been suspected to be a multiple system due to the discrepancy between the dynamically determined masses and the magnitude difference between stars A and B. The shape of the light curves in panels (d) and (f) of Fig. 5 is not consistent with a simple detached eclipsing binary, but it could be due to tidal distortions or backheating in a slightly eccentric binary system with a period of $11.6 \mathrm{~d}$. Indeed, Griffin (2004) points out that the period of a putative $85 \mathrm{Peg} \mathrm{Bb}$ of less than a few weeks would be possible.

Based on the location of $85 \mathrm{Peg} B$ in the HR diagram and by applying a mass-luminosity relation, D'Antona et al. (2005) suggested the luminosities of a possible secondary system to be $L_{\mathrm{Ba}} \sim 0.064 L_{\odot}$ and $L_{\mathrm{Bb}} \sim 0.0085 L_{\odot}$ (with the luminosity of the primary $L_{\mathrm{A}} \sim 0.617 L_{\odot}$ ). The masses of the components of the possible $85 \mathrm{Peg}$ Bab binary are estimated to be $M_{\mathrm{Ba}} \sim 0.5 M_{\odot}$ and $M_{\mathrm{Bb}} \sim 0.2 M_{\odot}$ (Bach et al. 2007; D'Antona et al. 2005). Therefore, an orbital period $P=11.6 \mathrm{~d}$ means a semi-major axis $a \sim 0.09 \mathrm{AU}$ (about $20 R_{\odot}$ ). If the stars in $85 \mathrm{Peg} \mathrm{B}$ are on the main sequence, then this separation would be about $20 \times$ the expected diameter of star $\mathrm{Ba}$.

The amplitude of the light variation is about $5 \mathrm{mmag}$ (allowing for dilution by star A), which means the 85 Peg Bab binary must produce a peak-to-peak variation of about $1 \%$. If the light variation is primarily a geometrical effect of tidal distortion, the light curve would be a double wave (with two maxima and two minima) during each binary orbit. The latter would mean the binary period would be $2 \times 11.6=23.2 \mathrm{~d}$. However, for such a period, the semi-major axis of the orbit would be $a \sim 0.14 \mathrm{AU}$, or about $30 \times$ the diameter of star Ba. At this separation, the geometrical distortion of star Ba by tides would be far below $1 \%$ and could not account for the full amplitude of the observed variation.

The bolometric flux on the surface of star Ba due to star $\mathrm{Bb}\left(L_{\mathrm{Bb}} \sim 0.0085 L_{\odot}\right.$ at a distance of about $\left.0.1 \mathrm{AU}\right)$ would be $\sim 1.5 \times 10^{4} \mathrm{~W} / \mathrm{m}^{2}$. The surface flux of star $\mathrm{Ba}\left(L_{\mathrm{Ba}} \sim 0.064 L_{\odot}\right.$; $R \sim 0.6 R_{\odot}$ ) would be roughly $1.4 \times 10^{8} \mathrm{~W} / \mathrm{m}^{2}$. Therefore the flux ratio due to backheating by a putative star $\mathrm{Bb}$ is only about $10^{-4}$, and the expected peak-to-peak amplitude would be in the order of $120 \mathrm{ppm}$. This is two orders of magnitude smaller than the observed peak variation in brightness, if that modulation originates in component B of the 85 Peg system.

Is it possible that the brightness ratio of stars $\mathrm{A}$ and $\mathrm{B}$ is not $\sim 16$ due to the custom design of the MOST bandpass, and that variability due to star B would not be diluted as much as we have assumed? We tested this possibility by generating stellar models across a range of masses, for ages of $8 \mathrm{Gyr}$, and determining their flux ratios measured through the MOST filter (Table 3).

To change the flux ratio measured by MOST significantly, the temperature of star A would have to be considerably cooler than measured for this relatively bright star. Even if the the ratio is lowered by a factor of 3 (last row of Table 3), the observed amplitude would still be (in the most optimistic case) by about a factor of 9 too low. Therefore, it is unlikely that illumination of a component $\mathrm{Ba}$ by a putative star Bb can easily account for the amplitude of the observed variation.

\subsection{Rotational modulation}

\subsubsection{Determination of rotational velocity}

In order to interpret the variation in terms of rotational modulation, a good knowledge of the projected rotational velocity $v \sin i$
Table 3. Model parameters for several possible configurations of the 85 Peg system and flux ratios as measured through the MOST custom filter.

\begin{tabular}{ccccccc}
\hline \hline Star & $\begin{array}{c}M \\
\left(M_{\odot}\right)\end{array}$ & $\begin{array}{c}R \\
\left(R_{\odot}\right)\end{array}$ & $\begin{array}{c}T_{\text {eff }} \\
(\mathrm{K})\end{array}$ & $\begin{array}{c}\Delta T^{i} \\
(\sigma)\end{array}$ & $\begin{array}{c}\text { Flux ratio } \\
\mathrm{A} / \mathrm{B}\end{array}$ & $\begin{array}{c}A_{\text {obs }}^{i i} \\
(\mathrm{mmag})\end{array}$ \\
\hline $\mathrm{A}$ & 0.90 & 0.92 & 5460 & 3 & 14.1 & 4.5 \\
$\mathrm{Ba}$ & 0.55 & 0.48 & 4200 & 0 & & \\
$\mathrm{Bb}$ & $\ldots$ & $\ldots$ & $\ldots$ & $\ldots$ & & \\
\hline $\mathrm{A}$ & 0.77 & 0.74 & 4845 & 15 & 1.5 & 0.48 \\
$\mathrm{Ba}$ & 0.72 & 0.68 & 4605 & 2 & & \\
$\mathrm{Bb}$ & $\ldots$ & $\ldots$ & $\ldots$ & $\ldots$ & & \\
\hline $\mathrm{A}$ & 0.90 & 0.92 & 5460 & 3 & 15.2 & 4.9 \\
$\mathrm{Ba}$ & 0.52 & 0.46 & 4160 & 0.2 & & \\
$\mathrm{Bb}$ & 0.20 & 0.21 & 3650 & $\ldots$ & & \\
\hline $\mathrm{A}$ & 0.77 & 0.74 & 4845 & 15 & 5.4 & \\
$\mathrm{Ba}$ & 0.52 & 0.46 & 4160 & 0.2 & & \\
$\mathrm{Bb}$ & 0.20 & 0.21 & 3650 & $\ldots$ & & \\
\hline
\end{tabular}

${ }^{i}$ The deviations in temperature to the observed value given in D'Antona et al. (2005) are given in terms of the standard deviation $\sigma$.

${ }^{i i} A_{\text {obs }}$ indicates the observed amplitude scaled with the corresponding flux ratio. The expected amplitude due to backheating of star $\mathrm{Bb}$ on star $\mathrm{Ba}$ is about $0.1-0.2 \mathrm{mmag}$.

is essential. Searching the literature we found measurements in the catalogues of Bernacca \& Perinotto (1970) and Uesugi \& Fukuda (1970) giving $v \sin i$ values of $3 \pm 3$ and $<6 \mathrm{~km} \mathrm{~s}^{-1}$, respectively, for $85 \mathrm{Peg}$ A. More recently, Wolff et al. (1985) measured $v \sin i \simeq 4 \mathrm{~km} \mathrm{~s}^{-1}$ and Hale (1994) gives a value of $1.8 \pm 0.6 \mathrm{~km} \mathrm{~s}^{-1}$. The discrepancy between all these measurements is evident, and hence $v \sin i$ of $85 \mathrm{Peg} \mathrm{A}$ remains a rather poorly determined parameter. Note that due to the close separation, no measurements for 85 Peg B are available.

With the goal to arrive at an independent $v \sin i$ measurement including statistically meaningful uncertainty estimates, we extracted high-resolution $(R \sim 60000)$ spectra taken with the UVES spectrograph from the ESO archive which were previously used to study star formation in the galaxy through abundance ratios (project number 076.B-0133).

After the pipeline reduction and continuum normalization, synthetic spectra were computed using the program synth3 (Kochukhov 2007) in conjunction with VALD (Piskunov et al. 1995; Kupka et al. 1999; Ryabchikova et al. 1999). The fitting of the syntheses to the observed $\mathrm{H} \alpha$ line, which can be seen in Fig. 6, resulted in $\log g=4.5$ and an effective temperature $T_{\text {eff }}=5500 \mathrm{~K}$.

Based on this effective temperature, MARCS model atmospheres (Gustafsson et al. 2008) were chosen for the subsequent analysis. After the equivalent widths were determined using a modified version (Tsymbal 1996) of the WIDTH9 code (Kurucz 1993), a microturbulence velocity $\left(v_{\text {mic }}\right)$ of $1.3 \mathrm{~km} \mathrm{~s}^{-1}$ was determined by eliminating any abundance-equivalent width correlation. Specific Fe lines with highly accurate $\log g_{\mathrm{f}}$ (Ryabchikova, private communication) were used for the $v \sin i$ determination. An Fe abundance of $-0.35 \pm 0.05$ dex relative to the solar value (Asplund et al. 2005) and a radial velocity of $34.6 \mathrm{~km} \mathrm{~s}^{-1}$ were adopted to enable a good comparison of observation and synthesis. Since the effects of macroturbulence velocity $\left(v_{\text {mac }}\right)$ and $v \sin i$ on the goodness-of-fit in the spectrum are indistinguishable to the naked eye (see Fig. 7), we pursued to approximate the marginal distribution of $v \sin i$.

For this purpose, we calculated a 2D-grid of synthetic spectra, using the parameter values mentioned above, but with $v \sin i$ 


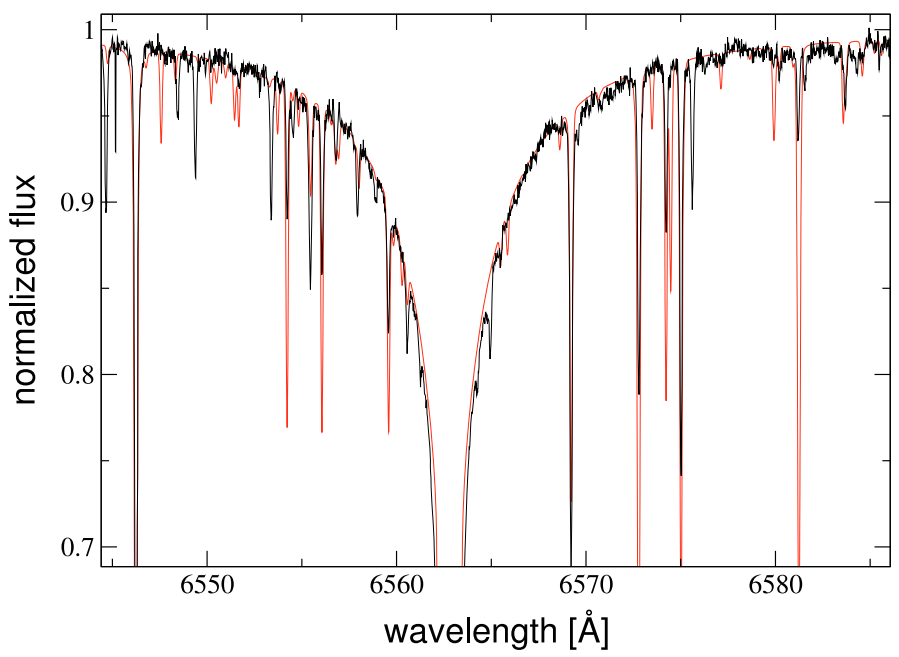

Fig. 6. Observed UVES spectrum (thick black line) and fitted synthetic spectrum with $\log g=4.5$ and $T_{\text {eff }}=5500 \mathrm{~K}$ (thin red line).

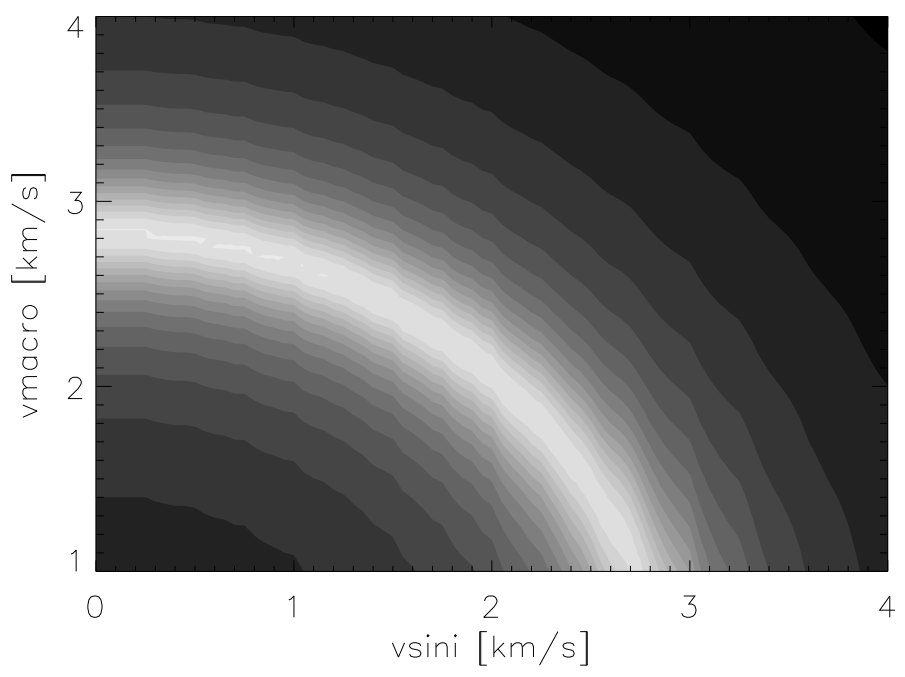

Fig. 7. The goodness-of-fit of observed to synthetic spectrum of one Fe line as a function of $v \sin i$ and $v_{\text {mac }}$. Bright areas indicate a good fit.

ranging from 0 to $4 \mathrm{~km} \mathrm{~s}^{-1}$ and $v_{\text {mac }}$ ranging from 1 to $4 \mathrm{~km} \mathrm{~s}^{-1}$ in steps of 0.05. Subsequently, we compared this grid of models to the four most clearly defined iron lines in the observed spectrum. However, each of these lines was treated as an independent observation to overall create four probability distributions. The error bars of each observed data point, as delivered by the reduction pipeline, were interpreted as $1 \sigma$-confidence limits and the probability for each model value was calculated according to

$p\left(d_{\mathrm{O}, i}\right)=\exp \left(-\frac{\left(d_{\mathrm{O}, i}-d_{\mathrm{M}, i}\right)^{2}}{2 \sigma_{i}^{2}}\right)$,

where $d_{\mathrm{O}, i}$ is the $i$ th observed data point, $d_{\mathrm{M}, i}$ is the corresponding model value, and $\sigma_{i}$ the aforementioned $1 \sigma$-uncertainty. The probability $p_{\mathrm{M}}$ for each model then is the product of the probabilities for all data points.

We calculated the marginal distributions of $v \sin i$ for each line by integrating out the $v_{\text {mac }}$ parameter. Since we used a discrete parameter space in our 2D-grid, we summed up the model probabilities over the whole parameter range of $v_{\mathrm{mac}}$ for each specific value of $v \sin i$, and normalized the distribution so that the sum of all resulting probabilities is unity. Finally, we combined the marginal distributions of all Fe lines by calculating the

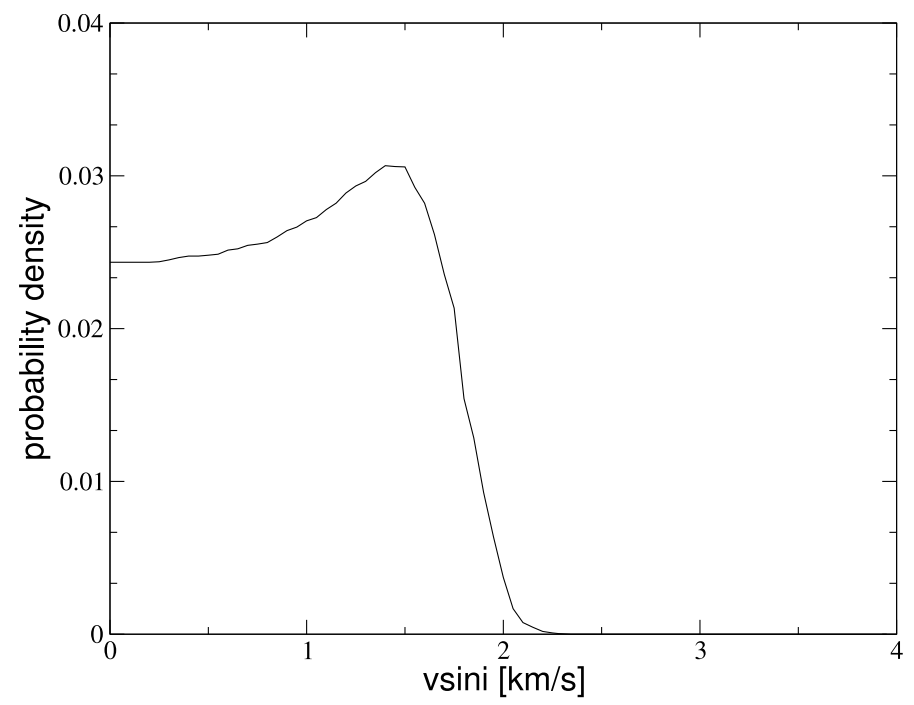

Fig. 8. The marginal distribution of $v \sin i$ for 85 Peg A derived as described in Sect. 4.2.1. There is no evidence for a $v \sin i$ above $2.5 \mathrm{~km} \mathrm{~s}^{-1}$ according to the UVES data we used for our analysis.

joint probabilities for all models and, again, normalized the resulting distribution. The final distribution is shown in Fig. 8. It was subsequently used as a prior ${ }^{3}$ for the $v \sin i$ parameter in the spot model analysis described in the next section. Judging from the distribution alone, we obtain $v \sin i=1.40_{-1.10}^{+0.15}$ (with the uncertainties roughly corresponding to a $1 \sigma$ confidence limit).

\subsubsection{Spot model fitting}

To test the possibility of rotational modulation, we fit the 2005 and 2007 light curves (panels (d) and (f) of Fig. 5) with simple spot models. We employ the StarSpotz functionality discussed in Croll et al. (2006a) and Croll (2006), which adopts circular spots and applies analytic models described by Budding (1977). StarSpotz has been used to measure the differential rotation profile of another MOST target, the young active solar-type star $\kappa^{1}$ Ceti (Walker et al. 2007) and it was able to account for the photometric variations detected by MOST and constrain the differential rotation coefficient of the exoplanet-hosting star $\epsilon$ Eri (Croll et al. 2006b). The reader is reminded at this point that in the following modeling efforts we explicitly assume that the variation seen in panel (d) of Fig. 5 is intrinsic, and disregard evidence for more complex variability as seen in panel (e) of Fig. 5.

A separate spot model was generated for components A and B of the 85 Peg system. Linear limb darkening coefficients for both stars were estimated using the closest grid points to the physical parameters from D'Antona et al. (2005) in the catalog of Claret (2000); these were $u \sim 0.86$ for 85 Peg A and $u \sim 0.80$ for $85 \mathrm{Peg} \mathrm{B}$. Note that considering the uncertainty of the observed variation and the fact that we are only intending to provide a first approximation to model the observed variation, we refrain from using a more complex limb-darkening model in this application. We used a flux contrast between the spotted and unspotted photosphere of $\kappa_{w}=0.30$ for $85 \mathrm{Peg} \mathrm{A}$ and $\kappa_{w}=0.35$

3 In Bayesian probability analysis, the prior is a probability distribution conveying already existing knowledge about a quantity. The information contained in the prior is used to constrain and assess the final probability of the outcome (see Bayesian inference textbooks for details). 

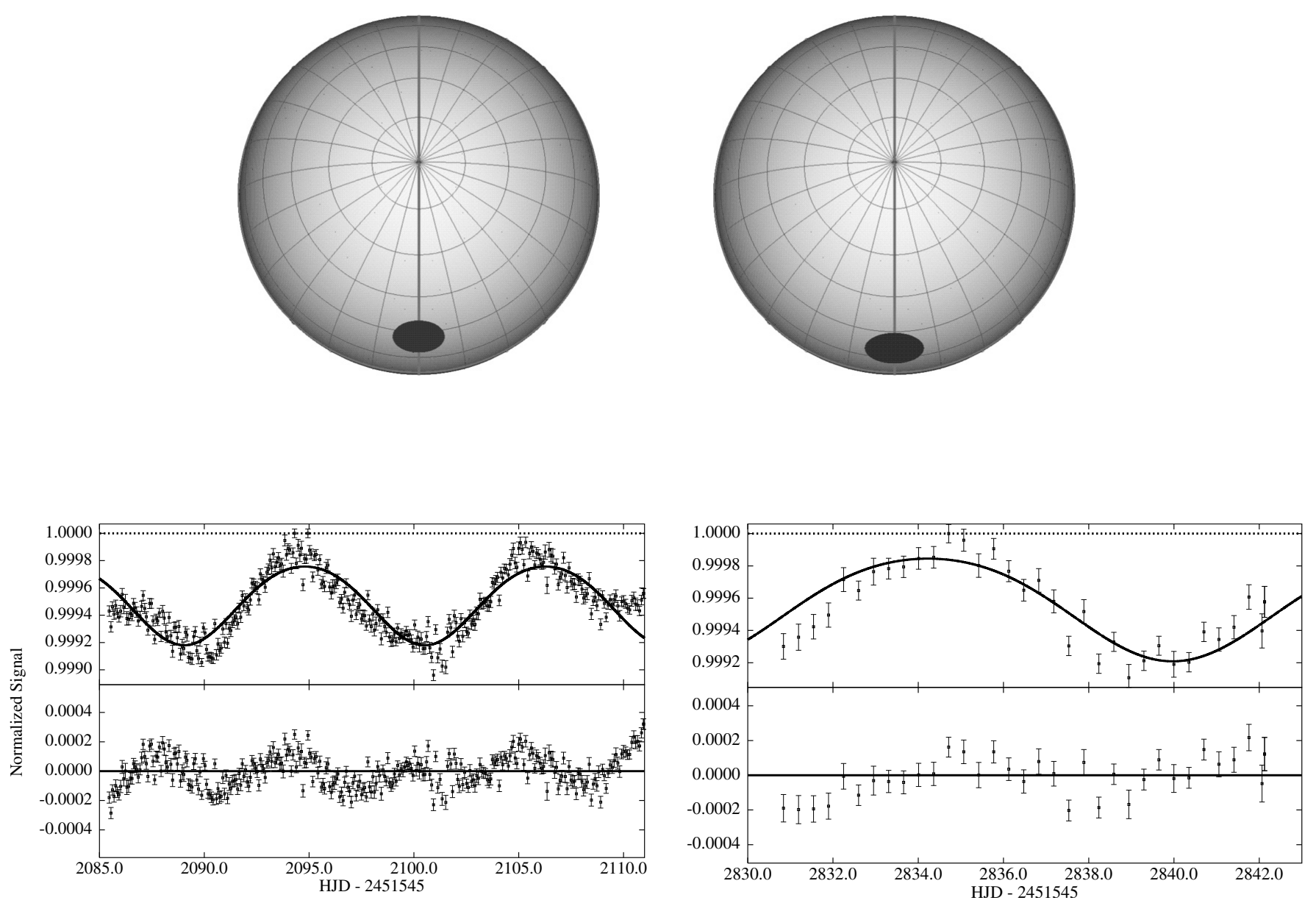

Fig. 9. Spot models of the periodic light variation observed by MOST in the 85 Peg system if caused by 85 Peg B. Top: the best fitting one-spot configurations for 85 Peg B with the spot facing the viewer for 2005 (left) and 2007 (right). Bottom left: the top panel shows the observed (binned) 2005 light curve and the model fit for $85 \mathrm{Peg} \mathrm{B}$, while the bottom panel displays the residuals to the fit. Bottom right: same as the middle panel except for the 2007 light curve.

for $85 \mathrm{Peg} \mathrm{B}$. We are unable to independently determine $\kappa_{w}$, and small variations in $\kappa_{w}$ will only serve to decrease or increase the size of the modeled spots. The value for $85 \mathrm{Peg} \mathrm{A}$ is appropriate for a G5IV star as it is comparable to that of the Sun and for the value chosen for $\kappa^{1}$ Ceti (Walker et al. 2007). We assumed a similar value for the K6-8V star 85 Peg B. For the 85 Peg B model, the light curve was scaled to an amplitude of $5 \mathrm{mmag}$ to allow for the dilution by star A. For our 85 Peg A spot-model fit we place a priori constraint on the resulting $v \sin i$ value (thus a joint prior information on the rotation period and the rotational inclination angle of the star) based on the values calculated in Sect. 4.2.1. For $85 \mathrm{Peg} B$ we do not use such a prior constraint since the $v \sin i$ value of this target is unknown.

If the modulation is due to a single spot rotating on star A, the near-sinusoidal shape and low amplitude $(0.3 \mathrm{mmag})$ of the light curves require this spot to be small (for the adopted flux contrast) and for it to be visible for the duration of the light curve. Our determination of $v \sin i$ rules out edge-on and near edge-on inclinations for $85 \mathrm{Peg} \mathrm{A}$; however, the uncertainty in $v \sin i$ is still significant and thus our spot models of this star could vary from near pole-on inclination angles to much more moderate values $\left(i \sim 30^{\circ}\right)$. Also, the observed modulation could be due to many spots or more complicated spot geometries. The spot model that follows should thus be in no way considered unique, but simply an example of a possible one-spot spot model that could be causing the observed modulation.
The best model for star A using the 2005 data features a spot of diameter $2^{\circ}$ at a latitude of about $50^{\circ}$, while the 2007 data features a spot of diameter $2^{\circ}$ at a latitude of about $46^{\circ}$ relative to the equator. Our spot model has a rotational inclination angle of $i \sim 21^{\circ}$. Note that since changing the inclination angle of 85 Peg A does not significantly affect the $\chi^{2}$ of the spot model, our best fit model has an inclination angle that results in a $v \sin i$ similar to the mean of the distribution determined by our analysis in Sect. 4.2.1. For the radius of $R_{\mathrm{A}}=0.82 \pm 0.02 R_{\odot}$ - modeled by D'Antona et al. (2005) for the range of possible ages of $85 \mathrm{Peg} \mathrm{A}$ and close to the value of $0.846 R_{\odot}$ estimated by Fernandes et al. (2002) - and a rotation period $P=11.6 \mathrm{~d}$, the equatorial rotation velocity of the star would be $3.6 \mathrm{~km} \mathrm{~s}^{-1}$. The spot model inclination results in a value of $v \sin i$ of about $1.3 \mathrm{~km} \mathrm{~s}^{-1}$ (compared to our $v \sin i \sim 1.40_{-1.10}^{+0.15}$ value determined in Sect. 4.2.1).

For star B, the intrinsic amplitude of the light variation should be closer to $5 \mathrm{mmag}$, requiring a larger spot if the contrast ratio between the spotted and unspotted photosphere is similar to that of other K6-8V stars. The best-fit model is presented in Fig. 9 (note that the best-fit model for star A is very similar, except with a larger inclination angle and smaller spot diameter). In this case, the inclination of the star for the best fitted model is $i \simeq 11^{\circ}$. In 2005 the spot in the best-fit model has a diameter of $8^{\circ}$ at a latitude of $27^{\circ}$. In 2007 the spot has a diameter of $9^{\circ}$ at a latitude of $20^{\circ}$. The equatorial rotation velocity of star B 
would be about $4.7 \mathrm{~km} \mathrm{~s}^{-1}$ and the resulting $v \sin i$ of the model is about $0.9 \mathrm{~km} \mathrm{~s}^{-1}$. We could find no measurements of $v \sin i$ for 85 Peg B in the literature.

\section{Conclusions}

Nearly continuous photometry of the binary system 85 Peg obtained with the MOST space telescope in 2005 yields a null result for the detection of $\mathrm{p}$-modes in the metal-poor subdwarf 85 Peg A. Spanning over 25 days and reaching down to a noise level of about $4 \mathrm{ppm}$ in the frequency region of interest, the high precision data present the most complete photometry of the system collected so far and enable us to set an upper limit for the detection of such oscillations. Based on simulations of stochastically excited and damped p-mode signal, we conclude that the rms amplitude of oscillations in 85 Peg A must lie below $\sim 12-18 \mathrm{ppm}$ if the mode lifetimes are short (i.e. on the order of days) and below $\sim 10 \mathrm{ppm}$ if the mode lifetimes are long (i.e. on the order of weeks). Based on these results, we provide upper limits for the Lorentzian profile height and the bolometric amplitude per radial mode as a function of mode lifetime and find these limits to be in agreement with theoretical values. Due to the fact that the predicted amplitude is a factor 4-5 lower than the detection threshold, however, we are unable to comment further on the comparison of theoretical and observed amplitudes as recently done by Michel et al. (2008) for CoRoT observations.

We detect light variations in the 85 Peg system with a period of about $11 \mathrm{~d}$ and a peak amplitude of $0.3 \mathrm{mmag}$ in our 2005 epoch of data and confirm this variability in the 2007 epoch of data. However, as different methods to remove the instrumental background in the 2005 data yield vastly different results, we cannot exclude the possibility that we do not know the exact shape and period of the 2005 variability. Nevertheless, as the 2005 and 2007 data yield similar results we believe it is likely that 85 Peg displayed consistent variability over these two epochs.

The observed peak amplitude of the periodicity can in any case not be explained by effects of a possible undetected binary 85 Peg B. Ignoring correlations with telemetry data which exist but cannot be interpreted beyond doubt, we fitted simple models to the residual data under the assumption that the variation is caused by star spots modulated with the rotation period of one of the components. To facilitate the search for such models we analyzed high-resolution UVES spectra of 85 Peg found in the ESO archive and derived a new estimate of $v \sin i=1.40_{-1.10}^{+0.15}$ for 85 Peg A. While we are able to reproduce the light curves of both datasets well with single-spot models, it must be emphasized that the solutions presented here are by no means unique, and there is no evidence that rules out more complex models.

Assuming that our simple models are realistic, this would indicate a rotation period of $11.6 \mathrm{~d}$ for either one of the components. Noyes et al. (1984) derived a rotation period for 85 Peg A of 23.8 days indirectly based on its chromospheric activity index. The evolutionary models of D'Antona et al. (2005) allow ages from 8-14 Gyr, while those of Bach et al. (2007) result in an age of $8.4 \pm 0.5 \mathrm{Gyr}$ for both stars in the $85 \mathrm{Peg}$ system. Component B is expected to be coeval with star A and should share the same age. All of these age estimates suggest that the stars should have a slower rotation period than the observed variation shows. For example, the much younger active G5 dwarfs $\kappa^{1}$ Ceti (Walker et al. 2007) and HD 189733 (Henry \& Winn 2008) have rotation periods of 9 and 11.8 days, respectively. This is comparable to the 11.6 days rotation period suggested for $85 \mathrm{Peg}$ despite the fact that the 85 Peg system is much older and should have a much longer rotation period.

The spectroscopic binary solution summarized by Griffin (2004) yields an orbit inclination of $|i| \sim 50^{\circ}$. While we do not explicitly assume a spin-orbit coupling in the system to constrain the inclination in the spot models described above, it is worth noting that for $85 \mathrm{Peg} \mathrm{A}$, a rotation period of twice the observed period (23.2 d) with such an inclination would result in a $v \sin i$ of $1.4 \mathrm{~km} \mathrm{~s}^{-1}$, which is also consistent with the values derived in Sect. 4.2.1 and might be a more realistic rotation period (suggesting that indeed a more complex spot model is required to explain the variation). Another, maybe more exotic explanation for the short period might be that the rotational modulation is produced in star B (the cooler and potentially more active component) and that its rotation is synchronized with the long suspected binary companion with an orbital period of $11.6 \mathrm{~d}$.

Lastly, we strongly encourage follow-up observations to confirm the $11.6 \mathrm{~d}$ observed variability.

Acknowledgements. We are grateful for discussions with Pierre Demarque. We are also thankful to Gerald Handler for critical discussions concerning the p-mode detection limit. M.G., M.O., T.K., W.W.W. and R.K. are supported by the Austrian Fonds zur Förderung der wissenschaftlichen Forschung, project number P17580-N02. The Austrian participation in the MOST project is funded by the Austrian Research Promotion Agency (FFG). B.C., D.B.G., J.M.M., A.F.J.M., and S.R. acknowledge funding from the Natural Sciences \& Engineering Research Council (NSERC) Canada.

\section{References}

Allende Prieto, C., Barklem, P. S., Lambert, D. L., \& Cunha, K. 2004, A\&A, 420,183

Appourchaux, T., Michel, E., Auvergne, M., et al. 2008, A\&A, 488, 705

Asplund, M., Grevesse, N., \& Sauval, A. J. 2005, in Cosmic Abundances as Records of Stellar Evolution and Nucleosynthesis, ed. T. G. Barnes, III \& F. N. Bash, ASP Conf. Ser., 336, 25

Bach, K., Kim, Y.-C., \& Demarque, P. 2007, J. Astron. Space Sci., 24, 31

Baily, F. 1845, The catalogue of the British Association for the advancement of science (London: R. a. J. E. Taylor)

Barban, C., Matthews, J. M., de Ridder, J., et al. 2007, A\&A, 468, 1033

Baudin, F., Samadi, R., Goupil, M.-J., et al. 2005, A\&A, 433, 349

Bedding, T. R., Kjeldsen, H., Butler, R. P., et al. 2004, ApJ, 614, 380

Bedding, T. R., Butler, R. P., Carrier, F., et al. 2006, ApJ, 647, 558

Bernacca, P. L., \& Perinotto, M. 1970, Contributions dell'Osservatorio Astrofisica dell'Universita di Padova in Asiago, 239, 1

Bruntt, H. 2009, ArXiv e-prints

Bruntt, H., De Cat, P., \& Aerts, C. 2008, A\&A, 478, 487

Budding, E. 1977, Ap\&SS, 48, 249

Carrier, F., Kjeldsen, H., Bedding, T. R., et al. 2007, A\&A, 470, 1059

Chaplin, W. J., Elsworth, Y., Howe, R., et al. 1997, MNRAS, 287, 51

Chaplin, W. J., Houdek, G., Elsworth, Y., et al. 2005, MNRAS, 360, 859

Chaplin, W. J., Houdek, G., Karoff, C., Elsworth, Y., \& New, R. 2009, ArXiv e-prints

Claret, A. 2000, VizieR Online Data Catalog, 336, 31081

Croll, B. 2006, PASP, 118, 1351

Croll, B., Walker, G. A., Kuschnig, R., et al. 2006a, in BAAS, 38, 1217

Croll, B., Walker, G. A. H., Kuschnig, R., et al. 2006b, ApJ, 648, 607

D’Antona, F., Cardini, D., Di Mauro, M. P., et al. 2005, MNRAS, 363, 847

Fernandes, J., Morel, P., \& Lebreton, Y. 2002, A\&A, 392, 529

Fulbright, J. P. 2000, AJ, 120, 1841

Griffin, R. F. 2004, The Observatory, 124, 429

Guenther, D. B., Demarque, P., Kim, Y.-C., \& Pinsonneault, M. H. 1992, ApJ, 387,372

Guenther, D. B., Kallinger, T., Gruberbauer, M., et al. 2008, ApJ, 687, 1448

Gustafsson, B., Edvardsson, B., Eriksson, K., et al. 2008, A\&A, 486, 951

Hale, A. 1994, AJ, 107, 306

Hall, Jr., R. G. 1948, AJ, 54, 102

Henry, G. W., \& Winn, J. N. 2008, AJ, 135, 68

Holmberg, J., Nordström, B., \& Andersen, J. 2007, A\&A, 475, 519

Houdek, G. 2006, in ESA SP, 624, Proceedings of SOHO 18/GONG

2006/HELAS I, Beyond the spherical Sun

Huber, D., \& Reegen, P. 2008, Commun. Asteroseismol., 152, 77 
Kallinger, T., Gruberbauer, M., Guenther, D. B., Fossati, L., \& Weiss, W. W. 2008a, ArXiv e-prints

Kallinger, T., Guenther, D. B., Matthews, J. M., et al. 2008b, A\&A, 478, 497

Kjeldsen, H., \& Bedding, T. R. 1995, A\&A, 293, 87

Kochukhov, O. P. 2007, in Physics of Magnetic Stars, 109

Kupka, F., Piskunov, N., Ryabchikova, T. A., Stempels, H. C., \& Weiss, W. W. 1999, A\&AS, 138, 119

Kurucz, R. 1993, ATLAS9 Stellar Atmosphere Programs and 2 km/s grid. Kurucz CD-ROM No. 13, Cambridge, Mass.: Smithsonian Astrophysical Observatory, 13

Matthews, J. M. 2007, Commun. Asteroseismol., 150, 333

Michel, E., Baglin, A., Auvergne, M., et al. 2008, Science, 322, 558

Michel, E., Samadi, R., Baudin, F., et al. 2009, A\&A, 495, 979

Mosser, B., Bouchy, F., Catala, C., et al. 2005, A\&A, 431, L13

Mosser, B., Deheuvels, S., Michel, E., et al. 2008, A\&A, 488, 635

Noyes, R. W., Hartmann, L. W., Baliunas, S. L., Duncan, D. K., \& Vaughan, A. H. 1984, ApJ, 279, 763

Piskunov, N. E., Kupka, F., Ryabchikova, T. A., Weiss, W. W., \& Jeffery, C. S. 1995, A\&AS, 112, 525
Reegen, P. 2007, A\&A, 467, 1353

Reegen, P., Kallinger, T., Frast, D., et al. 2006, MNRAS, 367, 1417

Rowe, J. F., Matthews, J. M., Kuschnig, R., et al. 2006, Mem. Soc. Astron. Ital., 77,282

Ryabchikova, T., Piskunov, N., Savanov, I., Kupka, F., \& Malanushenko, V. 1999, A\&A, 343, 229

Ryabchikova, T., Fossati, L., \& Shulyak, D. 2009, ArXiv e-prints

Samadi, R., Georgobiani, D., Trampedach, R., et al. 2007, A\&A, 463, 297

Stello, D., Kjeldsen, H., Bedding, T. R., \& Buzasi, D. 2006, A\&A, 448, 709

ten Brummelaar, T., Mason, B. D., McAlister, H. A., et al. 2000, AJ, 119, 2403

Tsymbal, V. 1996, in M.A.S.S., Model Atmospheres and Spectrum Synthesis, ed. S. J. Adelman, F. Kupka, \& W. W. Weiss, ASP Conf. Ser., 108, 198

Uesugi, A., \& Fukuda, I. 1970, Catalogue of rotational velocities of the stars, Contributions from the Institute of Astrophysics and Kwasan Observatory, University of Kyoto, Kyoto: University, Kwasan Observatory, Institute of Astrophysics

Walker, G., Matthews, J., Kuschnig, R., et al. 2003, PASP, 115, 1023

Walker, G. A. H., Croll, B., Kuschnig, R., et al. 2007, ApJ, 659, 1611

Wolff, S. C., Heasley, J. N., \& Varsik, J. 1985, PASP, 97, 707 